\title{
The current therapeutic scenario for relapsed mantle cell lymphoma
}

\author{
Simone Ferrero ${ }^{\mathrm{a}, \mathrm{b}}$ and Martin Dreyling ${ }^{\mathrm{a}}$
}

\begin{abstract}
Purpose of review
Patients with relapsing mantle cell lymphoma (MCL) still represent a demanding challenge for the hematooncologist. The dismal prognosis and the absence of generally accepted therapeutic standards hamper the clinical management of such cases. Moreover, the availability of many targeted approaches, in a field so far missing efficient salvage regimens, challenges current therapeutic algorithms in these patients.

Recent findings

Molecular targeted drugs provide unprecedented response rates in relapsed and even chemorefractory MCL. Many phase II studies demonstrated impressive antilymphoma activity of compounds such as bortezomib, lenalidomide and temsirolimus, whereas ongoing phase III trials currently assess the 'real world' benefit and the impact on survival, both alone and in combination with chemotherapy or monoclonal antibodies. Recently, the Bruton's tyrosine kinase inhibitor ibrutinib, targeting the B-cell receptor cascade, showed impressive response rates and will be soon available in phase III trials.
\end{abstract}

\section{Summary}

In the present review we focus on the major therapeutic discoveries of the last few years to offer a practical algorithm to select the appropriate treatment in patients with relapsed $\mathrm{MCL}$.

\section{Keywords}

mantle cell lymphoma, new drugs, refractory, relapse, therapy

\section{INTRODUCTION}

During the last few years remarkable progress has been made in the development of new treatment strategies of mantle cell lymphoma (MCL), an infrequent subtype of non-Hodgkin lymphoma characterized by initial high responses but continuous relapse pattern and a dismal long-term outcome [1]. Most of the new targeted approaches available in the clinics or being tested in early phase I/II protocols result from recent molecular studies on cell proliferation and apoptosis pathways [2]. Moreover, ongoing molecular genetic investigations continue to identify new oncogenic targets, providing preclinical rationale for therapeutic application and further drugs development [3-5]. Nevertheless, a definite cure for MCL cannot be achieved so far, with the exception of potentially harmful allogeneic stem cell transplantation (allo-SCT). Even more important a satisfactory disease control can be achieved only in a subset of patients, particularly in relapsed disease.

The major clinical trials of the last decade focused on improvement of the front-line treatment, leading to the definition of a 'gold standard' therapy, for young and fit patients consisting of anthracyclines, high doses of cytarabine and rituximab, followed by an autologous stem cell transplantation (auto-SCT) [6-8,9"]. Similar efficiency has been shown for an even more intensified regime [10]; however, only the minority of patients was able to complete the full course in a multicenter setting [11].

Similarly the standard first-line therapy for elderly MCL patients (not eligible for auto-SCT) has been lately established consisting of rituximabcyclophosphamide-doxorubicin-vincristine-prednisone (R-CHOP) immunochemotherapy, followed by

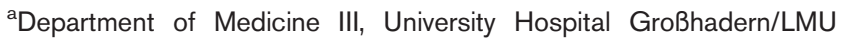

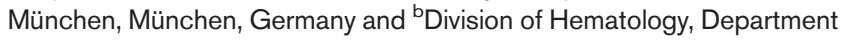
of Molecular Biotechnologies and Health Sciences, University of Torino, Torino, Italy

Correspondence to Professor Dr Martin Dreyling Medizinische Klinik und Poliklinik III Klinikum der Universität München, Marchioninistrasse 15, 81377 München, Germany. Tel: +49 897095 2202; fax: +49 897095 2201; e-mail: Martin.Dreyling@med.uni-muenchen.de
}

Curr Opin Oncol 2013, 25:452-462

DOI:10.1097/CCO.0b013e328363dfOb 


\section{KEY POINTS}

- The major biological discoveries of the last years in MCL survival pathways have led to the availability in the clinics of many effective targeted drugs.

- Phase II and III clinical trials are currently ongoing to test the efficacy of these drugs on large series of relapsed $M C L$ patients.

- Clinical trials investigating innovative approaches should be considered in all relapsed MCL patients.

- The treatment strategies in relapsed $M C L$ should depend on the individual risk profile and patients' comorbidities.

rituximab maintenance [12]. Both efforts resulted in a considerable improvement in overall survival.

On the contrary, lacking large randomized clinical trials, there is still no generally accepted approach in relapsed MCL. Clinical guidelines, although recently updated [9"], are not specifically focused on the relapse setting, supplying only some hints. Moreover, the numerous new targeted options [2], along with the promising data for combinations with conventional chemotherapeutics and anti-CD20 immunotherapy, could generate hesitation about the optimal therapeutic approach for these patients. Thus, the present review focuses on the major therapeutic discoveries of the last few years to offer a practical algorithm to select appropriate treatment decisions in patients with relapsed MCL.

\section{CURRENT AVAILABLE THERAPEUTIC OPTIONS}

As no curative treatment can be so far offered to relapsed MCL patients, aside from allo-SCT, the therapeutic goal should be the prolonged disease control, balancing the expected efficacy with the risk of toxicity and reduced quality of life (QoL). In this perspective the most recent data on salvage regimens are discussed below.

\section{Chemotherapy-based approaches}

The addition of rituximab to conventional chemotherapy (R-FCM, R-GEMOX, R-DHAP) increases response rates up to $60-70 \%$; however, the duration of response in relapsing disease remains limited (mainly less than 1 year) and such treatment options should be considered basically palliative [13-15]. More promising data on the contrary have been recently achieved from the new combination of some 'old drugs' with rituximab, based on the results of the bendamustin-rituximab regimen (75\% overall response rate, ORR - and 50\% complete responses, CR - in relapsing disease) [16]. The impressive activity of bendamustin-rituximab has been also shown in first-line treatment [17], as well as the superior data of cytarabine in first-line young patients [6-8]. Accordingly, a new regimen combining rituximab, bendamustine and cytarabine (R-BAC) was tested in 20 relapsed and 20 first diagnosed MCL patients [18"]. The activity of this combination is noteworthy, although in a limited series: an ORR of 80 with $70 \%$ CR was reported in relapsing patients (90 and $83 \%$ on the total, respectively), resulting in an excellent 2-year progression-free survival (PFS) of 70\% (95\% for the untreated patients). The primary toxicity was reversible myelosuppression, platelet transfusions were required in two-third of cycles and erythropoietin was applied in about half of patients. Currently, a phase II study of R-BAC in first-line for elderly patients is ongoing, but this regimen is promising also in the relapse setting.

In contrast, considering the low efficacy and the increased toxicity of an auto-SCT approach in second-line [19], such a treatment should be offered only to fit MCL patients who did not receive auto-SCT as first-line. Finally, among palliative approaches for elderly patients, the efficacy, feasibility and low toxicity of an oral low-dose metronomic polichemotherapy combination (PEP-C) is noteworthy, optionally in combination with rituximab and thalidomide $[20,21]$.

\section{Established molecular approaches}

In this section the most recent data on salvage 'new drugs' regimens are extensively discussed.

\section{Proteasome inhibitors}

The first 'new drug' to be registered in relapsed MCL in the United States is bortezomib, since the first demonstrations of this selective and reversible proteasome 26S inhibitor's efficacy [22-25]. Even though the combination of bortezomib with rituximab and chemotherapy showed high response rates (up to 60-70\%), the majority of these studies consists of small series of heavily pretreated patients and often comprises other histologies. Median PFS rates are in the range of 12 months. The published combined regimens encompass both targeted approaches, such as rituximab or rituximab-dexamethasone [26,27], as well as immunochemotherapies such as rituximabdexamethasone-high dose-cytarabin (R-HAD), rituximab-prednisone-cyclophosphamide (R-CP), rituximab-bendamustine and gemcitabine [28-31]. The 
toxicity profile predominantly consists of polyneuropathy (sometimes marked and long lasting) and neutrothrombocytopenia (when associated with chemotherapy), challenging the long-term application, for example, as maintenance in relapsed MCL. A phase III clinical trial is currently ongoing, randomizing MCL relapsed patients to receive either $\mathrm{R}-\mathrm{HAD} \pm$ bortezomib (NCT01449344). Other studies tested bortezomib combinations in first-line treatment, too [32-34].

\section{Mammalian target of rapamycin inhibitors}

Temsirolimus, an intravenous mammalian target of rapamycin (mTOR) inhibitor, received European Medicines Agency approval in 2009, due to its single-agent activity in patients with relapsed MCL. This approval was based on results of a large phase III trial in patients with relapsed/refractory MCL. Temsirolimus induced a significant improvement in median PFS and ORR, compared with investigator's choice monotherapy (4.8 versus 1.9 months and 22 versus $2 \%$, respectively) [35]. Hematological adverse events were the most frequently reported, but were generally well managed by dose reductions or treatment delay. Gastrointestinal toxicity, especially diarrhea, and fatigue were also common, but incidence of grade 3-4 events was low. The addition of rituximab to temsirolimus was subsequently tested in a phase II study on 71 patients. An increased ORR of 59\%, with up to $19 \%$ CR was observed, with a median time to progression (TTP) of about 10 months [36]; the toxicity profile was similar to temsirolimus monotherapy, with a slightly higher rate of pulmonary toxicity (pneumonia and pneumonitis around 10\% of cases). To further improve its efficacy, temsirolimus is being currently investigated in combination with bendamustin-rituximab in a phase II clinical trial (NCT01078142) [37].

Another well tolerated oral mTOR inhibitor is everolimus. In a multicenter phase II trial of 35 MCL-relapsed patients a ORR of $20 \%$ (with $49 \%$ of stable diseases, SD) with a median PFS of 5.5 months has been reported [38], but further studies in combination with chemotherapy or other biological drugs are warranted.

\section{Immunomodulatory drugs}

A number of phase II trials have confirmed the promising response rates of lenalidomide in relapsed MCL. This immunomodulatory compound showed high antilymphoma activity in several other studies [39-41]. The ORR were around 30-50\%, with promising CR rates up to $20 \%$ and PFS generally around 6-9 months. Recently a phase II study in
52 patients with relapsed MCL confirmed the impact of a chemo-free lenalidomide-rituximab combination with high response rates (57\% ORR, $36 \% \mathrm{CR}$ ) and impressive response durations up to 19 months [42"']. On the other hand, no clear advantages in survival were achieved by the addition of dexamethasone to lenalidomide [43]. The manageable toxicity (mainly mild, hematological) and the oral formulation make this drug an attractive option also in the context of maintenance regimens, specifically in the elderly population. A phase II trial of the rituximab-lenalidomide-bendamustine combination is presently accruing patients with relapsed MCL (NCT01737177).

Largely overshadowed by its subsequent follower lenalidomide, thalidomide, the oldest member of the immunomodulatory drugs (IMIDs), was shown active in relapsed MCL already a decade ago [44]. More recently, a retrospective French survey on 58 patients confirmed that thalidomide was effective in relapsed MCL, with a favorable side-effect profile (7\% grade 3-4 adverse events, including thromboembolism). Although this survey comprised different treatment schedules (monotherapy or combinations with rituximab or bortezomib), an interesting ORR of $50 \%$ (and 29\% SD) with a time to treatment failure (TTF) of 29 and $11 \%$ at 1 and 2 years, respectively, should be noticed [45]. Thus, thalidomide is well tolerated and might offer a cost-effective alternative to more expensive targeted agents, especially in countries with limited health-care resources.

\section{Antibody-based approaches}

In contrast to its favorable safety profile (mainly manageable thrombocytopenia and neutropenia) and promising data coming in other types of lymphoma, yttrium-90 $\quad\left({ }^{90} \mathrm{Y}\right)$-ibritumomab tiuxetan monotherapy does not impact substantially the prognosis of relapsed MCL. In a phase II trial of 32 patients with relapsed MCL this anti-CD20 radio-immunoconjugate showed an ORR of 32\% with an event-free survival (EFS) of 6 months [46]. However, radioimmunotherapy might be more efficient as part of multimodal strategies: considering that the results were particularly poor in patients with bulky disease, recent studies are exploring the ${ }^{90} \mathrm{Y}$-ibritumomab tiuxetan consolidation after successful salvage chemotherapy or first-line treatment [47] and the combination with another efficient targeted drug, such as bortezomib [48].

\section{NEW MOLECULAR-TARGETED APPROACHES}

The growing insights into the underlying molecular biology of MCL form the basis for the ongoing 
exploration of targeted approaches [2]. A number of new compounds are currently being tested in MCL and are available for application within clinical trials.

\section{B-cell receptor signaling inhibitors}

The most convincing data come for the oral Bruton's tyrosine kinase (BTK) inhibitor ibrutinib. The interim results of an international phase II trial on refractory/relapsed MCL patients show impressive efficacy and excellent tolerability of this drug, which specifically blocks the B-cell receptor (BCR) signaling survival pathway [49"']. In 110 evaluable patients (either bortezomib exposed or naive) ibrutinib monotherapy displayed an impressive ORR of $68 \%$, with $22 \%$ CR; the data are even superior focusing on the initial cohort of 51 patients, with a longer follow-up of nearly 15 months. ORR and CR were 75 and 39\%, respectively, clearly demonstrating an incremental CR rate under continuous treatment. Median duration of response is not yet reached with a PFS of around 14 months. Noteworthy is the favorable safety profile, with less than $15 \%$ grade $3 / 4$ hematological toxicity and mainly mild gastrointestinal symptoms, fatigue and infections in a population of heavily pretreated patients. These outstanding results have already led to the design of phase III trials, comparing ibrutinib versus temsirolimus in relapsed patients (NCT01646021) or conventional immunochemotherapy \pm ibrutinib as first-line treatment (www.clinicaltrials.gov). Moreover, the preclinical rationale for BTK-inhibitors combinations with other biological agents (such as bortezomib) has been already postulated [50].

Another disruptor of the BCR signal cascade, CAL-101, a specific inhibitor of phosphatidylinositol 3-kinase delta isoform, is currently being tested in phase I/II trials: although achieving a promising ORR of $62 \%$ [51], mature results on remission duration are not yet available.

\section{Antibody-based approaches}

New monoclonal antibodies $(\mathrm{mAB})$, targeting a variety of epitopes in addition to CD20 are currently investigated in preclinical and clinical trials, but data on MCL are still scarce. GA101 (obinutuzu$\mathrm{mab})$, the first type II, glycoengineered and humanized anti-CD20 mAB, determined a ORR of $27 \%$ (four out of 15 patients) in a randomized phase II trial of refractory/relapsed MCL [52]. Ofatumumab, a fully human $\mathrm{mAB}$ targeting a unique epitope on the CD20 molecule, has been tested in phase I/II trials in combination with bendamustine [53] or lenalidomide [54], but more mature data are still missing.

An interesting approach is the bispecific antiCD19/anti-CD3 mAB, which showed a high efficacy in a phase I/II trial particularly in the MCL patients $[55,56]$.

DCDS4501A is an anti-CD79b mAB conjugated to a microtubule toxin (monomethyl auristatin E), showing an acceptable toxicity profile and encouraging antitumor activity in a phase I trial on 33 heavily pretreated lymphoma patients, including four patients with relapsed/refractory MCL [57]. Nevertheless, additional studies on larger patient cohorts are warranted.

\section{Cell cycle/apoptosis targeting drugs and others}

Flavopiridol directly inhibits cyclin-dependent kinase (CDK) 4 and 6, leading to downregulation of cyclin D1. This compound showed significant activity in combination with fludarabine and rituximab or bortezomib in two phase I trials [58,59]. A direct CDK4/6 inhibitor, PD0332991, also achieved substantial responses and suggested clinical benefit in a subset of MCL patients [60].

ABT-199 is an orally bioavailable, second-generation BCL-2 specific BH3 mimetic with promising results in a phase I trial for MCL patients (six out of six patients with a $>50 \%$ reduction in target lesions) [61].

Abexinostat, a novel oral pan histone deacetylase inhibitor, proved to be clinically active and well tolerated in a phase II trial in patients with refractory/relapsed MCL. ORR was 27\% (three out of 11 patients) and PFS 4 months. The most common grade $3 / 4$ adverse events were thrombocytopenia $(17 \%)$, neutropenia (13\%), fatigue (13\%) and anemia $(7 \%)$ [62].

A summary of the recent published clinical trials of targeted approaches in relapsed MCL is presented in Table 1.

\section{ALLOGENEIC TRANSPLANTATION}

Despite the high response rates obtained by the new therapeutic approaches so far described, a long-term disease control has not yet been achieved in relapsed MCL and prognosis of subsequent relapses remains poor, in particular in chemorefractory cases. The only few reported definite long-term remissions (or even cure) for at least a selected subset of relapsed patients are achieved by allo-SCT. The biological background of such an immunologic approach is based on a lymphoma-free graft as well as the supposed allogeneic reactivity of the donor T-cells 


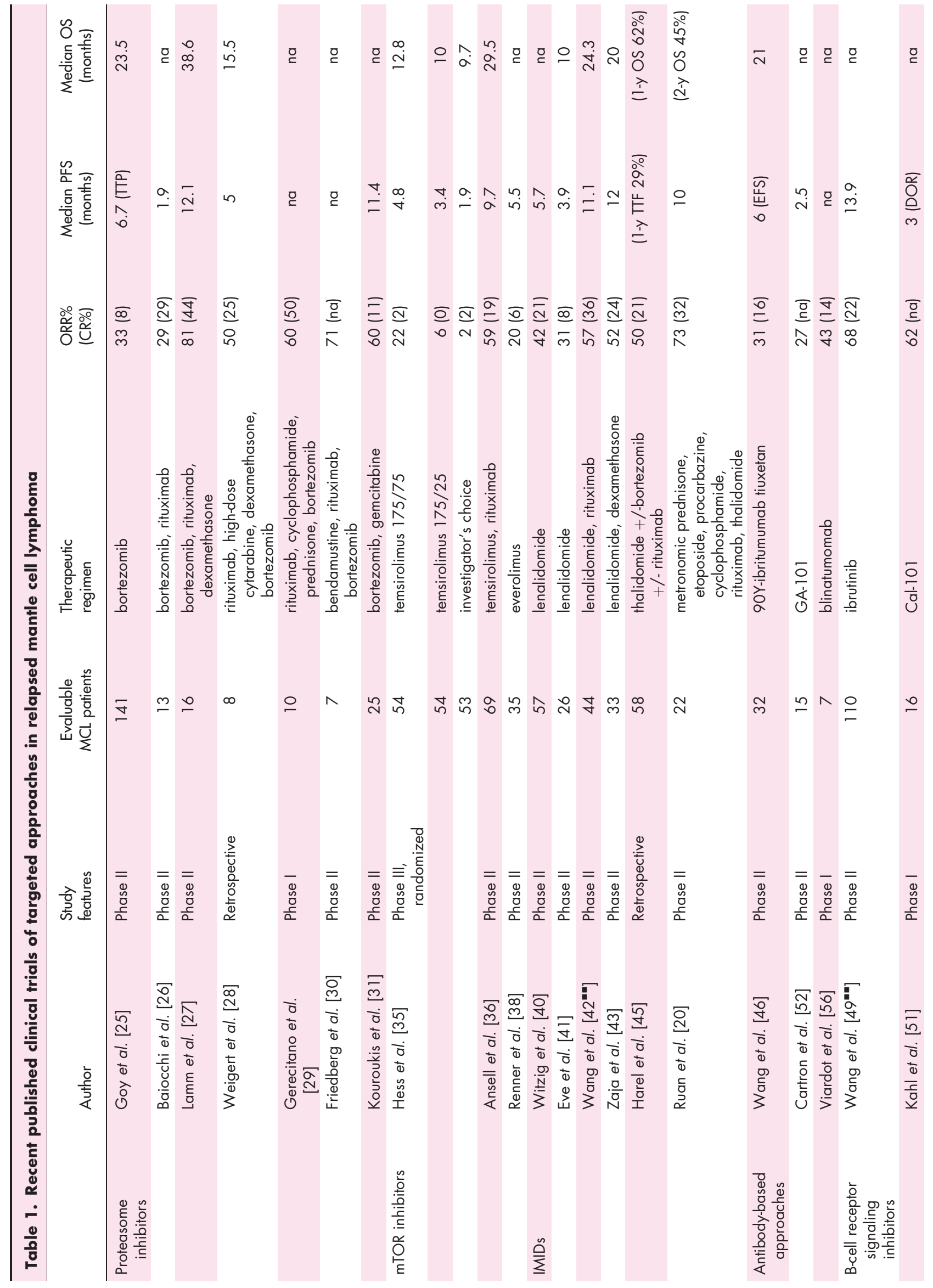




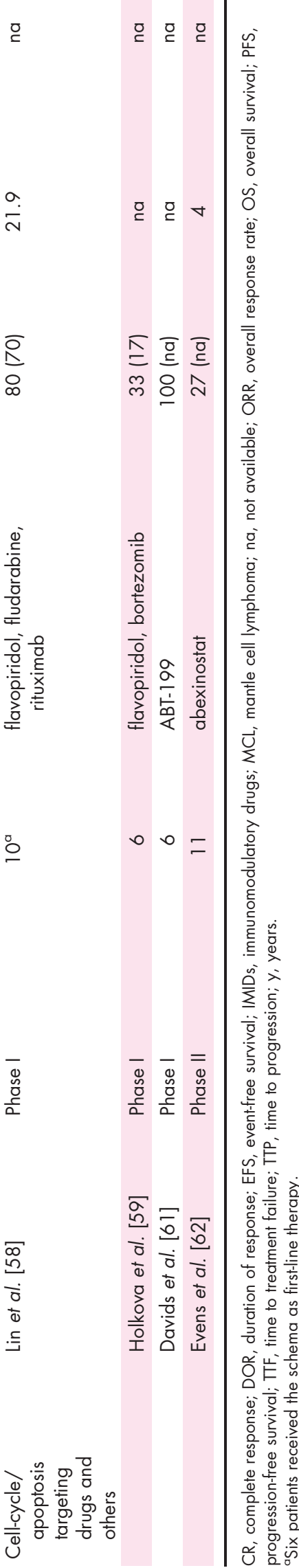

against the tumor, a phenomenon known as 'graftversus-lymphoma' reaction, potentially overcoming also chemoresistance. However, the application of allo-SCT in MCL is hampered by the therapy-related high toxicity of both reduced-intensity conditioning (RIC) and nonmyeloablative (NMA) approaches. Moreover, limitations come from advanced patient age (median at diagnosis is 65-68 years), availability of a compatible donor and the high relapse rate even after this procedure.

In the retrospective single center experience of M.D. Anderson [19] 35 most-chemosensitive (88\%) patients (median age 58 years) received a NMA alloSCT (fludarabine, cyclophosphamide and high-dose rituximab). A 6-year PFS rate of $46 \%$ was reported, with a plateau phase after 3 years and no relapse after 63 months of follow-up, whereas the 6-year overall survival (OS) was 53\%. The 1-year treatmentrelated mortality (TRM) rate was $9 \%$ but no longterm nonrelapse mortality (NRM) rate was stated.

A recent retrospective multicenter French report [63] assessed the outcome of 70 MCL patients (79\% chemosensitive, median age 56 years) receiving a RIC allo-SCT following various regimens (94\% fludarabine-containing). In this series, the 2-year EFS and OS rates were 50 and 53\%, respectively, but no plateau phase was achieved at any time point after transplantation. The 1-year and 2-year TRM rates were 22 and $32 \%$, respectively.

Interestingly, in a recent large retrospective study of 202 patients with chemorefractory MCL, the Center for International Blood and Marrow Transplant Research found no significant differences between patients receiving conventional and RIC/ NMA allo-SCT in terms of 3-year PFS (20 versus 25\%), OS (25 versus 30\%) and NRM (47 versus 43\%) [64"].

In conclusion, although the discussed studies may be hampered by selection bias in single center series, heterogeneity of patients and procedures, it is noteworthy that an allo-SCT approach achieves durable remissions in approximately a fourth of young chemorefractory patients [64"]. The addition of radioimmunotherapy (RIT) to allo-SCT procedures has shown only conflicting results so far $[65,66]$. In conclusion, the issue of tolerability of such an immunological strategy is essential, as the high NRM (along with the high relapse rate) remains the major limitation for the application of allo-SCT in relapsed MCL.

\section{ONGOING EUROPEAN CLINICAL TRIALS FOR RELAPSED MANTLE CELL LYMPHOMA}

As MCL is a rare entity and relapsed MCL patients are difficult to treat with conventional immunochemotherapy, an enrollment in a prospective clinical 
Table 2. Ongoing European clinical trials for relapsed mantle cell lymphoma

\begin{tabular}{|c|c|c|c|c|c|c|}
\hline NCT code & Study features & $\begin{array}{l}\text { Estimated } \\
\text { enrollment } \\
\text { (patients) }\end{array}$ & $\begin{array}{c}\text { Estimated primary } \\
\text { completion date } \\
\text { (month/year) }\end{array}$ & $\begin{array}{l}\text { Therapeutic } \\
\text { regimen }\end{array}$ & Sponsor & $\begin{array}{l}\text { Location } \\
\text { countries }\end{array}$ \\
\hline 01449344 & $\begin{array}{l}\text { Phase III, } \\
\text { randomized }\end{array}$ & 157 & $09 / 2016$ & $\begin{array}{l}\text { R-HAD versus } \\
\text { R-HADB }\end{array}$ & European MCL Network & $\begin{array}{l}\text { France, } \\
\text { Germany }\end{array}$ \\
\hline 01646021 & $\begin{array}{l}\text { Phase III, } \\
\text { randomized }\end{array}$ & 280 & $08 / 2014$ & $\begin{array}{l}\text { Ibrutinib versus } \\
\text { temsirolimus }\end{array}$ & $\begin{array}{l}\text { Janssen Research \& } \\
\text { Development LLC }\end{array}$ & Worldwide \\
\hline 01078142 & $\begin{array}{l}\text { Phase } I / I I \text {, single } \\
\text { arm }\end{array}$ & 72 & $03 / 2014$ & BERT & $\begin{array}{l}\text { German Low Grade } \\
\text { Lymphoma Study } \\
\text { Group (GLSG) \& } \\
\text { European MCL } \\
\text { Network }\end{array}$ & Germany \\
\hline 01737177 & $\begin{array}{l}\text { Phase II, single } \\
\text { arm }\end{array}$ & 42 & $07 / 2014$ & $\mathrm{R}-2 \mathrm{~B}$ & $\begin{array}{l}\text { Fondazione Italiana } \\
\text { Linfomi ONLUS, Italy }\end{array}$ & Italy \\
\hline 01389427 & $\begin{array}{l}\text { Phase I/II, } \\
\text { single group } \\
\text { assignment }\end{array}$ & 63 & $06 / 2013$ & $\begin{array}{l}\text { R-CHOP/R-FC/ } \\
\text { R-HAD }+ \\
\text { temsirolimus }\end{array}$ & $\begin{array}{l}\text { Groupe Ouest Est } \\
\text { d'Etude des } \\
\text { Leucémies et Autres } \\
\text { Maladies du Sang } \\
\text { GOELAMS, France }\end{array}$ & France \\
\hline
\end{tabular}

BERT, bendamustine-rituximab-temsirolimus; $M C L$, mantle cell lymphoma; $N C T$, national clinical trial; $R-H A D \pm B$, rituximab high-dose cytarabine-dexamethasone \pm bortezomib; R-2B, rituximab-lenalidomide-bendamustine; R-CHOP, rituximab-cyclophosphamide-doxorubicin-vincristine-prednisone; R-FC, rituximab-fludarabinecyclophosphamide. Details of the studies can be found at the internet site: http://www.clinicaltrials.gov

trial is strongly recommended. This option allows applying new effective and expensive medications, sometimes not yet exploitable in routine clinical practice. Thus, a list of the major European actively recruiting clinical trials for relapsed MCL is reported in Table 2. The details of each study can be found at: http://www.clinicaltrials.gov

\section{Prospective, multicenter, randomized, open- label phase III clinical trials}

'Efficacy and Safety of R-HAD Alone or in Combination With Bortezomib in Patients With Relapsed or Refractory MCL' (NCT01449344), compares the efficacy and safety of bortezomib in combination with rituximab, high-dose cytarbine and dexamethasone (R-HAD) to R-HAD alone in patients with relapsed or refractory MCL after or not eligible for myeloablative treatment. Estimated enrollment: 200 patients. Sponsor: European MCL Network.

'Study of Ibrutinib (a Bruton's Tyrosine Kinase Inhibitor), Versus Temsirolimus in Patients With Relapsed or Refractory Mantle Cell Lymphoma Who Have Received at Least One Prior Therapy' (NCT01646021), evaluates the efficacy and safety of ibrutinib when compared with temsirolimus in patients with relapsed or refractory MCL who have received at least one prior rituximab-containing chemotherapy regimen. Estimated enrollment: 280 patients. Sponsor: Janssen Research \& Development LLC.

\section{Prospective, multicenter, single arm, open- label phase II or I/II clinical trials}

'Temsirolimus, Bendamustine and Rituximab for Relapsed Follicular Lymphoma or Mantle Cell Lymphoma' (NCT01078142): the efficacy of the combination regimens will be evaluated in a first cohort of 30 patients with relapsed MCL and in a second cohort of 30 patients with relapsed follicular lymphoma. Estimated enrollment: 72 patients. Sponsor: German Low Grade Lymphoma Study Group (GLSG) and European MCL Network.

'Bendamustine/Lenalidomide/Rituximab: Combination as a Second-Line Therapy for First Relapsed-Refractory MCL' (NCT01737177), evaluates the safety and activity of the combination of bendamustine, lenalidomide and rituximab (R2-B) in patients with first relapsed/refractory MCL and the efficacy and safety of a maintenance treatment with lenalidomide for 18 months from the end of R2-B. Estimated enrollment: 42 patients. Sponsor: Fondazione Italiana Linfomi ONLUS, Italy.

'Escalating Doses of Torisel in Combination With Three Chemotherapies Regimens: R-CHOP, R-FC or R-DHA for Patients With Relapsed/Refractory MCL' (NCT01389427), assesses the feasibility, safety and efficacy of temsirolimus in combination with three chemotherapy regimens. Estimated enrollment: 63 patients. Sponsor: Groupe Ouest Est d'Etude des Leucémies et Autres Maladies du Sang GOELAMS, France. 


\section{CONCLUSION}

Considering the complexities in the treatment of relapsed MCL, these patients should be generally referred to experienced centers to determine the optimal therapeutic strategy and potential inclusion into a clinical trial. In general treatment strategies should depend on the individual risk profile and patients' comorbidities. Our brief practical suggestions how to treat a patient with relapsed MCL are based on the most recent literature, evidence-based guidelines [9"] and clinical experience (Fig. 1).

For young ( $<65$ years) and fit patients allo-SCT should be considered in all cases after appropriate first-line therapy, due to its curative potential. The salvage regimen should contain cytarabine or bendamustine and rituximab in combination, when possible, with a targeted approach such as bortezomib, lenalidomide or temsirolimus (R-BAC,
R-HADB, BERT, R-2B regimens). The major goal is the achievement of at least a partial response, although a fraction of chemorefractory patients may obtain prolonged remissions, too. For patients not eligible for allo-SCT it is crucial to implement additional consolidation concepts (e.g. rituximab maintenance, RIT consolidation, new molecules within studies) to maintain remission.

For elderly ( $>65$ years) fit patients, young nonfit patients or patients relapsing after allo-SCT a treatment with curative intent is not established. A salvage regimen containing targeted approaches is strongly recommended and the enrollment into a clinical trial is advisable. A tailored therapy should be based on individual risk profile, for example, elevated Ki67 levels [67] may favor a combination of cytarabine and bortezomib (R-HADB) or plus bendamustine (R-BAC) for fit patients, whereas a regimen with bendamustine-rituximab

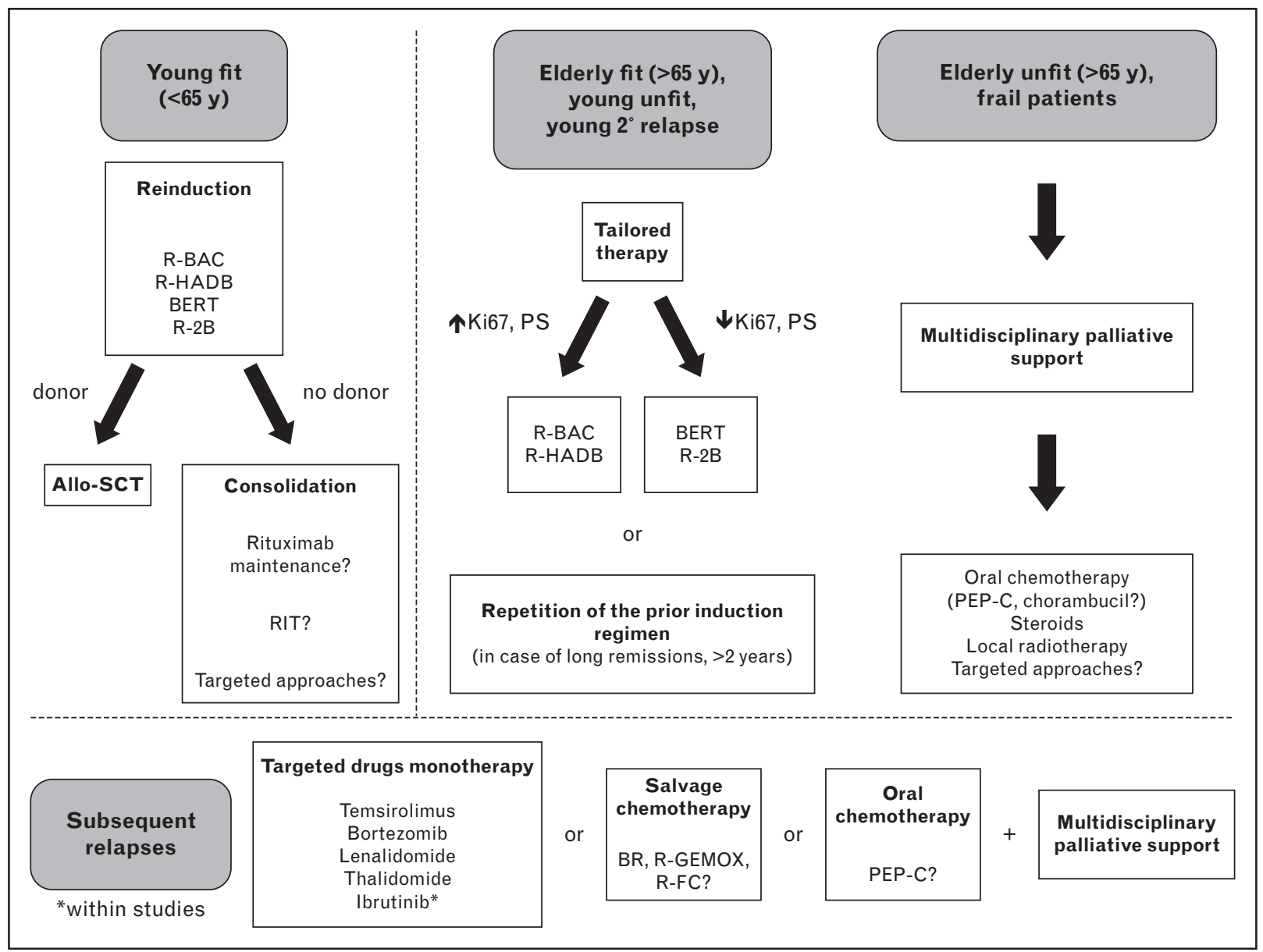

FIGURE 1. A rational therapeutic algorithm for patients with relapsed MCL. allo-SCT, allogeneic stem cell transplantation; auto-SCT, autologous stem cell transplantation; BERT, bendamustine-rituximab-temsirolimus; Ki67, Ki67 proliferative index; PEP$C$, metronomic prednisone-etoposide-procarbazine-cyclophosphamide; PS, performance status; R-BAC, rituximab-bendamustinecytarabine; R-HADB, rituximab high-dose cytarabine-dexamethasone-bortezomib; R-2B, rituximab-lenalidomide-bendamustine; RIT, radioimmunotherapy; BR, bendamustine-rituximab; R-GEMOX, rituximab-gemcitabine-oxaliplatin; R-FC, rituximabfludarabine-cyclophosphamide; $y$, years. 
in combination with temsirolimus (BERT) or lenalidomide (R-2B) appears more suitable for less fit patients with a lower proliferation index. The repetition of the prior induction regimen could be an appropriate approach, if a longstanding remission ( $>2$ years) was previously achieved.

For subsequent relapses, along with the deterioration of performance status and QoL, monotherapies with targeted drugs (in particular ibrutinib in the context of a clinical trial, or bortezomib, lenalidomide, thalidomide, temsirolimus) or well tolerated combinations with rituximab, steroids or lowdose chemotherapy should be preferred. Oral palliative combinations, such as the metronomic PEP-C, could be also useful options in this setting. In addition, a multidisciplinary palliative support may be started.

For elderly unfit or frail relapsed patients the preservation of the QoL should be the primary objective of the clinical care. Thus, mild oral chemotherapy combinations, steroids, radiotherapy and in selected cases also molecular approaches represent the standard of care. An adequate palliative support is crucial.

\section{Acknowledgements}

This work was supported by Progetto di Ricerca Sanitaria Finalizzata 2008 (head unit: IRCCS Centro di Riferimento Oncologico della Basilicata (CROB), Rionero in Vulture (Potenza), Italy) (code: 7.07.08.60 P49), (head unit: Divisione di Ematologia, A. O. S. Maurizio, Bolzano/Bozen, Italy - code: 7.07.08.60 P51), Progetto di Ricerca Sanitaria Finalizzata 2009, (head unit: Divisione di Ematologia, A. O. S. Maurizio, Bolzano/Bozen, Italy - code: RF-2009-1469205), Progetto di Ricerca Sanitaria Finalizzata 2010, (head unit: Divisione di Ematologia, A. O. S. Maurizio, Bolzano/Bozen, Italy code: RF-2010-2307262), Università degli Studi di Torino, Torino, Italy and by Fondazione Neoplasie del sangue (FO.NE. SA), Torino, Italy.

\section{Conflicts of interest}

Authors' Disclosure of Potential Conflicts of Interest: M.D.: scientific advisory board (Celgene, Jannsen, Pfizer), speakers honoraria (Celgene, Jannsen, Pfizer, Roche), support of ITS (Celgene, Jannsen, Mundipharma, Pfizer, Roche). S.F. has no conflicts of interest.

\section{REFERENCES AND RECOMIMENDED \\ READING}

Papers of particular interest, published within the annual period of review, have been highlighted as:

- of special interest

-1. of outstanding interest

Additional references related to this topic can also be found in the Current

World Literature section in this issue (pp. 576-577).

1. Herrmann A, Hoster $E$, Zwingers $T$, et al. Improvement of overall survival in advanced stage mantle cell lymphoma. J Clin Oncol 2009; 27:511-518; doi: 10.1200/JCO.2008.16.8435
2. Pérez-Galán $P$, Dreyling $M$, Wiestner $A$. Mantle cell lymphoma: biology, pathogenesis, and the molecular basis of treatment in the genomic era. Blood 2011; 117:26-38; doi: 10.1182/blood-2010-04-189977.

3. Kridel R, Meissner B, Rogic S, et al. Whole transcriptome sequencing reveals recurrent $\mathrm{NOTCH} 1$ mutations in mantle cell lymphoma. Blood 2012; 119:1963-1971; doi: 10.1182/blood-2011-11-391474.

4. Iqbal J, Shen $Y$, Liu $Y$, et al. Genome-wide miRNA profiling of mantle cell lymphoma reveals a distinct subgroup with poor prognosis. Blood 2012; 119:4939-4948; doi: 10.1182/blood-2011-07-370122.

5. Choi YJ, Li X, Hydbring $P$, et al. The requirement for cyclin $D$ function in tumor maintenance. Cancer Cell 2012; 22:438-451; doi: 10.1016/j.ccr.2012. 09.015.

6. Geisler $\mathrm{CH}$, Kolstad A, Laurell $\mathrm{A}$, et al. Long-term progression-free survival of mantle cell lymphoma after intensive front-line immunochemotherapy with in vivo-purged stem cell rescue: a nonrandomized phase 2 multicenter study by the Nordic Lymphoma Group. Blood 2008; 112:2687-2693; doi: 10.1182/ blood-2008-03-147025.

7. Hermine O, Hoster E, Walewski J, et al. Alternating Courses of $3 x \mathrm{CHOP}$ and 3x DHAP Plus Rituximab Followed by a High Dose ARA-C Containing Myeloablative Regimen and Autologous Stem Cell Transplantation (ASCT) Increases Overall Survival When Compared to 6 Courses of CHOP Plus Rituximab Followed by Myeloablative Radiochemotherapy and ASCT in Mantle Cell Lymphoma: Final Analysis of the MCL Younger Trial of the European Mantle Cell Lymphoma Network (MCL net). Blood (ASH Annual Meeting Abstracts) 2012; 120:151

8. Le Gouill S, Callanan M, Macintyre E, et al. Clinical, Metabolic and Molecular Responses After 4 Courses of R-DHAP and After Autologous Stem Cell Transplantation for Untreated Mantle Cell Lymphoma Patients Included in the LyMa Trial, a Lysa Study. Blood (ASH Annual Meeting Abstracts) 2012; 120:152.

9. Dreyling M, Thieblemont C, Gallamini A, et al. ESMO Consensus confer-

- ences: guidelines on malignant lymphoma. part 2: marginal zone lymphoma, mantle cell lymphoma, peripheral T-cell lymphoma. Ann Oncol 2013; 24:857-877; doi: 10.1093/annonc/mds643.

Updated guidelines for diagnosis, prognostication and treatment of MCL.

10. Romaguera JE, Fayad LE, Feng $L$, et al. Ten-year follow-up after intense chemoimmunotherapy with Rituximab-HyperCVAD alternating with Rituximab-high dose methotrexate/cytarabine (R-MA) and without stem cell transplantation in patients with untreated aggressive mantle cell lymphoma. Br J Haematol 2010; 150:200-208; doi: 10.1111/j.1365-2141.2010.08228.x.

11. Merli $F$, Luminari $S$, Ilariucci $F$, et al. Rituximab plus HyperCVAD alternating with high dose cytarabine and methotrexate for the initial treatment of patients with mantle cell lymphoma, a multicentre trial from Gruppo Italiano Studio Linfomi. Br J Haematol 2012; 156:346-353; doi: 10.1111/j.1365-2141. 2011.08958.x

12. Kluin-Nelemans HC, Hoster $E$, Hermine $O$, et al. Treatment of older patients with mantle-cell lymphoma. N Engl J Med 2012; 367:520-531; doi: 10. 1056/NEJMoa1200920.

13. Forstpointner $R$, Dreyling $M$, Repp $R$, et al. The addition of rituximab to a combination of fludarabine, cyclophosphamide, mitoxantrone (FCM) significantly increases the response rate and prolongs survival as compared with FCM alone in patients with relapsed and refractory follicular and mantle cell lymphomas: results of a prospective randomized study of the German LowGrade Lymphoma Study Group. Blood 2004; 104:3064-3071.

14. Rodríguez J, Gutierrez A, Palacios $A$, et al. Rituximab, gemcitabine and oxaliplatin: an effective regimen in patients with refractory and relapsing mantle cell lymphoma. Leuk Lymphoma 2007; 48:2172-2178.

15. Witzig TE, Geyer SM, Kurtin PJ, et al. Salvage chemotherapy with rituximab DHAP for relapsed non-Hodgkin lymphoma: a phase II trial in the North Central Cancer Treatment Group. Leuk Lymphoma 2008; 49:1074-1080; doi: 10.1080/10428190801993470.

16. Rummel MJ, Al-Batran SE, Kim SZ, et al. Bendamustine plus rituximab is effective and has a favorable toxicity profile in the treatment of mantle cell and low-grade non-Hodgkin's lymphoma. J Clin Oncol 2005; 23:33833389.

17. Rummel MJ, Niederle $N$, Maschmeyer G, et al. Bendamustine plus rituximab versus CHOP plus rituximab as first-line treatment for patients with indolent and mantle-cell lymphomas: an open-label, multicentre, randomised, phase 3 noninferiority trial. Lancet 2013. doi:pii: S0140-673661763-2. 10.1016/ S0140-6736(12)61763-2.

18. Visco $C$, Finotto $S$, Zambello $R$, et al. Combination of Rituximab, Bendamus-

- tine, and Cytarabine for Patients With Mantle-Cell Non-Hodgkin Lymphoma Ineligible for Intensive Regimens or Autologous Transplantation. J Clin Oncol 2013.

Although on a limited patients series, this phase $\mathrm{I} / \mathrm{Il}$ study addresses a highly promising therapeutic combination for relapsed MCL.

19. Tam CS, Bassett R, Ledesma $C$, et al. Mature results of the M. D. Anderson Cancer Center risk-adapted transplantation strategy in mantle cell lymphoma. Blood 2009; 113:4144-4152; doi: 10.1182/blood-200810-184200.

20. Ruan J, Martin $P$, Coleman $M$, et al. Durable responses with the metronomic rituximab and thalidomide plus prednisone, etoposide, procarbazine, and cyclophosphamide regimen in elderly patients with recurrent mantle cell lymphoma. Cancer 2010; 116:2655-2664; doi: 10.1002/cncr.25055. 
21. Coleman M, Ruan G, Elstrom RL, et al. Metronomic therapy for refractory/ relapsed lymphoma: the PEP-C low-dose oral combination chemotherapy regimen. Hematology 2012; 17 (Suppl 1):S90-S92; doi: 10.1179/ $102453312 \times 13336169155970$.

22. Goy $A$, Younes $A$, McLaughlin $P$, et al. Phase II study of proteasome inhibitor bortezomib in relapsed or refractory B-cell non-Hodgkin's lymphoma. J Clin Oncol 2005; 23:667-675.

23. O'Connor OA, Wright J, Moskowitz C, et al. Phase Il clinical experience with the novel proteasome inhibitor bortezomib in patients with indolent non-Hodgkin's lymphoma and mantle cell lymphoma. J Clin Oncol 2005; 23:676-684.

24. Fisher RI, Bernstein SH, Kahl BS, et al. Multicenter phase II study of bortezomib in patients with relapsed or refractory mantle cell lymphoma. J Clin Oncol 2006; 24:4867-4874.

25. Goy A, Bernstein $\mathrm{SH}, \mathrm{Kahl} B \mathrm{~S}$, et al. Bortezomib in patients with relapsed or refractory mantle cell lymphoma: updated time-to-event analyses of the multicenter phase 2 PINNACLE study. Ann Oncol 2009; 20:520-525; doi: $10.1093 / a n n o n c / m d n 656$.

26. Baiocchi RA, Alinari L, Lustberg ME, et al. Phase 2 trial of rituximab and bortezomib in patients with relapsed or refractory mantle cell and follicular lymphoma. Cancer 2011; 117:2442-2451.

27. Lamm W, Kaufmann $\mathrm{H}$, Raderer $\mathrm{M}$, et al. Bortezomib combined with rituximab and dexamethasone is an active regimen for patients with relapsed and chemotherapy-refractory mantle cell lymphoma. Haematologica 2011; 96:1008-1014; doi: 10.3324/haematol.2011.041392.

28. Weigert $O$, Weidmann $E$, Mueck $R$, et al. A novel regimen combining high dose cytarabine and bortezomib has activity in multiply relapsed and refractory mantle cell lymphoma - long-term results of a multicenter observation study. Leuk Lymphoma 2009; 50:716-722; doi: 10.1080/10428190902856790.

29. Gerecitano J, Portlock $C$, Hamlin $P$, et al. Phase I trial of weekly and twiceweekly bortezomib with rituximab, cyclophosphamide, and prednisone in relapsed or refractory non-Hodgkin lymphoma. Clin Cancer Res 2011; 17:2493-2501; doi: 10.1158/1078-0432.CCR-10-1498.

30. Friedberg JW, Vose JM, Kelly JL, et al. The combination of bendamustine, bortezomib, and rituximab for patients with relapsed/refractory indolent and mantle cell non-Hodgkin lymphoma. Blood 2011; 117:2807-2812; doi: 10.1182/blood-2010-11-314708.

31. Kouroukis CT, Fernandez LA, Crump M, et al. A phase II study of bortezomib and gemcitabine in relapsed mantle cell lymphoma from the National Cancer Institute of Canada Clinical Trials Group (IND 172). Leuk Lymphoma 2011; 52:394-399; doi: 10.3109/10428194.2010.546015.

32. Ruan J, Martin P, Furman RR, et al. Bortezomib plus CHOP-rituximab for previously untreated diffuse large B-cell lymphoma and mantle cell lymphoma. J Clin Oncol 2011; 29:690-697; doi: 10.1200/JCO.2010.31.1142.

33. Houot R, Le Gouill S, Ojeda Uribe M, et al. Combination of rituximab, bortezomib, doxorubicin, dexamethasone and chlorambucil (RiPAD+C) as first-line therapy for elderly mantle cell lymphoma patients: results of a phase II trial from the GOELAMS. Ann Oncol 2012; 23:1555-1561; doi: 10.1093/ annonc/mdr450.

34. Kahl BS, Li H, Smith MR, et al. Mature Results From ECOG Study E1405 - A Phase II Study of VcR-CVAD with Maintenance Rituximab for Previously Untreated Mantle Cell Lymphoma. Blood (ASH Annual Meeting Abstracts) 2012; 120:153.

35. Hess G, Herbrecht R, Romaguera J, et al. Phase III study to evaluate temsirolimus compared with investigator's choice therapy for the treatment of relapsed or refractory mantle cell lymphoma. J Clin Oncol 2009; 27:3822 3829; doi: 10.1200/JCO.2008.20.7977.

36. Ansell $\mathrm{SM}$, Tang $\mathrm{H}, \mathrm{Kurtin} \mathrm{PJ}$, et al. Temsirolimus and rituximab in patients with relapsed or refractory mantle cell lymphoma: a phase 2 study. Lancet Oncol 2011; 12:361-368. doi: 10.1016/S1470-2045(11)70062-6.

37. Hess G, Keller H, Atta J, et al. Temsirolimus in Combination with Bendamustine and Rituximab for the Treatment of Relapsed Mantle Cell and Follicular Lymphoma: Report on An Ongoing Phase I/II Trial. Blood (ASH Annual Meeting Abstracts) 2011; 118:2697.

38. Renner C, Zinzani PL, Gressin R, et al. A multicenter phase II trial (SAKK 36/ 06) of single-agent everolimus (RAD001) in patients with relapsed or refractory mantle cell lymphoma. Haematologica 2012; 97:1085-1091; doi: 10.3324/haematol.2011.053173.

39. Wiernik PH, Lossos IS, Tuscano JM, et al. Lenalidomide monotherapy in relapsed or refractory aggressive non-Hodgkin's lymphoma. J Clin Oncol 2008; 26:4952-4957; doi: 10.1200/JCO.2007.15.3429

40. Witzig TE, Vose JM, Zinzani PL, et al. An international phase II trial of singleagent lenalidomide for relapsed or refractory aggressive B-cell non-Hodgkin's lymphoma. Ann Oncol 2011; 22:1622-1627; doi: 10.1093/annonc/ mdq626.

41. Eve HE, Carey S, Richardson SJ, et al. Single-agent lenalidomide in relapsed/ refractory mantle cell lymphoma: results from a UK phase II study suggest activity and possible gender differences. Br J Haematol 2012; 159:154-163; doi: $10.1111 /$ bjh.12008

42. Wang $\mathrm{M}$, Fayad L, Wagner-Bartak $\mathrm{N}$, et al. Lenalidomide in combination with

n rituximab for patients with relapsed or refractory mantle-cell lymphoma: a phase 1/2 clinical trial. Lancet Oncol 2012; 13:716-723. doi: 10.1016/ S1470-2045(12)70200-0.

The combination of lenalidomide and rituximab in 44 patients achieved the highest response and survival rates published so far for relapsed/refractory $\mathrm{MCL}$.
43. Zaja $F$, De Luca $S$, Vitolo $U$, et al. Salvage treatment with lenalidomide and dexamethasone in relapsed/refractory mantle cell lymphoma: clinical results and effects on microenvironment and neo-angiogenic biomarkers. Haematologica 2012; 97:416-422; doi: 10.3324/haematol.2011. 051813.

44. Kaufmann $\mathrm{H}$, Raderer $\mathrm{M}$, Wöhrer $\mathrm{S}$, et al. Antitumor activity of rituximab plus thalidomide in patients with relapsed/refractory mantle cell lymphoma. Blood 2004; 104:2269-2271.

45. Harel S, Bachy E, Haioun C, et al. Efficacy and Safety of Thalidomide In Mantle Cell Lymphoma: Results of the French ATU Program. Blood (ASH Annual Meeting Abstracts) 2010; 116:1794.

46. Wang $\mathrm{M}$, Oki $\mathrm{Y}$, Pro $\mathrm{B}$, et al. Phase II study of yttrium-90-ibritumomab tiuxetan in patients with relapsed or refractory mantle cell lymphoma. J Clin Oncol 2009; 27:5213-5218; doi: 10.1200/JCO.2009.21.8545

47. Smith MR, Li H, Gordon L, et al. Phase II study of rituximab plus cyclophosphamide, doxorubicin, vincristine, and prednisone immunochemotherapy followed by yttrium-90-ibritumomab tiuxetan in untreated mantle-cell lymphoma: Eastern Cooperative Oncology Group Study E1499. J Clin Oncol 2012; 30:3119-3126; doi: 10.1200/JCO.2012.42.2444

48. Beaven AW, Shea TC, Moore DT, et al. A phase I study evaluating ibritu-

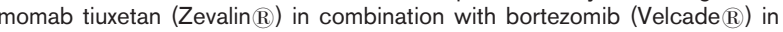
relapsed/refractory mantle cell and low grade B-cell non-Hodgkin lymphoma. Leuk Lymphoma 2012; 53:254-258; doi: 10.3109/10428194.2011. 608445. Epub 2011 Sep 19.

49. Wang $M$, Rule SA, Martin $P$, et al. Interim Results of an International, Multi-

1. center, Phase 2 Study of Bruton's Tyrosine Kinase (BTK) Inhibitor, Ibrutinib (PCl-32765), in Relapsed or Refractory Mantle Cell Lymphoma (MCL): Durable Efficacy and Tolerability with Longer Follow-up. Blood (ASH Annual Meeting Abstracts) 2012; 120:904

Although this abstract shows only the interim results of a large international phase II trial on 110 refractory/relapsed MCL patients, the high efficacy and the excellent tolerability of this drug are impressive.

50. Dasmahapatra G, Patel H, Dent $P$, et al. The Bruton tyrosine kinase (BTK) inhibitor PCl-32765 synergistically increases proteasome inhibitor activity in diffuse large-B cell lymphoma (DLBCL) and mantle cell lymphoma (MCL) cells sensitive or resistant to bortezomib. Br J Haematol 2013; 161:43-56; doi: 10.1111/bjh.12206.

51. Kahl B, Byrd JC, Flinn IW, et al. Clinical Safety and Activity In a Phase 1 Study of CAL-101, An Isoform-Selective Inhibitor of Phosphatidylinositol 3-Kinase P110\{delta\}, In Patients with Relapsed or Refractory Non-Hodgkin Lymphoma. Blood (ASH Annual Meeting Abstracts) 2010; 116:1777.

52. Cartron $G$, Thieblemont $C$, Solal-Celigny $P$, et al. Promising Efficacy with the New Anti-CD20 Antibody GA101 In Heavily Pre-Treated NHL Patients - First Results From a Phase II Study In Patients with Relapsed/Refractory DLBCL and MCL. Blood (ASH Annual Meeting Abstracts) 2010; 116:2878.

53. Magni M, Di Nicola M, Carlo-Stella C, et al. Safety, Tolerability and Activity of Ofatumumab, Bendamustine and Dexamethasone Combination As First-Line Treatment of Mantle-Cell Lymphoma in the Elderly: A Multicenter Study. Blood (ASH Annual Meeting Abstracts) 2011; 118:1647.

54. Vose JM, Loberiza FR, Bociek RG, et al. Phase I/II Trial of Ofatumumab/ Lenalidamide for Patients with Relasped/Refractory B-Cell Non-Hodgkin Lymphoma: High Response Rate in Indolent Lymphoma. Blood (ASH Annual Meeting Abstracts) 2012; 120:3692.

55. Bargou R, Leo E, Zugmaier G, et al. Tumor regression in cancer patients by very low doses of a T cell-engaging antibody. Science 2008; 321:974-977; doi: 10.1126/science.1158545.

56. Viardot A, Goebeler M, Scheele JS, et al. Treatment of Patients with Non-Hodgkin Lymphoma (NHL) with CD19/CD3 Bispecific Antibody Blinatumomab (MT103): Double-Step Dose Increase to Continuous Infusion of $60 \mu \mathrm{g} / \mathrm{m} 2 / \mathrm{d}$ ls Tolerable and Highly Effective. Blood (ASH Annual Meeting Abstracts) 2010; 116:2880.

57. Palanca-Wessels MC, Flinn IW, Sehn LH. e al. A Phase I Study of the AntiCD79b Antibody-Drug Conjugate (ADC) DCDS4501A Targeting CD79b in Relapsed or Refractory B-Cell Non-Hodgkin's Lymphoma (NHL). Blood (ASH Annual Meeting Abstracts) 2012; 120:56.

58. Lin TS, Blum KA, Fischer DB, et al. Flavopiridol, fludarabine, and rituximab in mantle cell lymphoma and indolent B-cell lymphoproliferative disorders. J Clin Oncol 2010; 28:418-423; doi: 10.1200/JCO.2009.24.1570.

59. Holkova B, Perkins EB, Ramakrishnan V, et al. Phase I trial of bortezomib (PS-341; NSC 681239) and alvocidib (flavopiridol; NSC 649890) in patients with recurrent or refractory B-cell neoplasms. Clin Cancer Res 2011; 17:3388-3397; doi: 10.1158/1078-0432.CCR-10-2876.

60. Leonard JP, LaCasce AS, Smith MR, et al. Selective CDK4/6 inhibition with tumor responses by PD0332991 in patients with mantle cell lymphoma. Blood 2012; 119:4597-4607; doi: 10.1182/blood-2011-10388298.

61. Davids MS, Roberts AW, Anderson MA, et al. The BCL-2-Specific BH3Mimetic ABT-199 (GDC-0199) Is Active and Well Tolerated in Patients with Relapsed Non-Hodgkin Lymphoma: Interim Results of a Phase I Study. Blood (ASH Annual Meeting Abstracts) 2012; 120:304.

62. Evens AM, Vose JM, Harb W, et al. A Phase II Multicenter Study of the Histone Deacetylase Inhibitor (HDACi) Abexinostat (PCl-24781) in Relapsed/Refractory Follicular Lymphoma (FL) and Mantle Cell Lymphoma (MCL). Blood (ASH Annual Meeting Abstracts) 2012; 120:55. 
63. Le Gouill S, Kröger N, Dhedin N, et al. Reduced-intensity conditioning allogeneic stem cell transplantation for relapsed/refractory mantle cell lymphoma: a multicenter experience. Ann Oncol 2012; 23:2695-2703.

64. Hamadani M, Saber W, Ahn KW, et al. Allogeneic hematopoietic cell

- transplantation for chemotherapy-unresponsive mantle cell lymphoma: a cohort analysis from the center for international blood and marrow transplant research. Biol Blood Marrow Transplant 2013; 19:625-631; doi: 10.1016/ j.bbmt.2013.01.009.

This large retrospective study of 202 patients with chemorefractory MCL receiving allogeneic transplantation indicates that durable remissions can be achieved at least in a fraction of these poor prognosis patients.
65. Bethge WA, von Harsdorf $\mathrm{S}$, Bornhauser $\mathrm{M}$, et al. Dose-escalated radioimmunotherapy as part of reduced intensity conditioning for allogeneic transplantation in patients with advanced high-grade non-Hodgkin lymphoma. Bone Marrow Transplant 2012; 47:1397-1402; doi: 10.1038/bmt.2012.62.

66. Gopal AK, Guthrie KA, Rajendran J, et al. ${ }^{90} \mathrm{Y}$-lbritumomab tiuxetan, fludarabine, and TBI-based nonmyeloablative allogeneic transplantation conditioning for patients with persistent high-risk B-cell lymphoma. Blood 2011; 118:1132-1139; doi: 10.1182/blood-2010-12-324392.

67. Hoster E, Klapper W, Rosenwald A, et al. Cell Proliferation (Ki-67) As Prognostic Marker in Mantle Cell Lymphoma. Blood (ASH Annual Meeting Abstracts) 2012; 120:2677. 